\title{
Nationalization of innovation Wuqiao Acrobatics garment
}

\author{
QiaoNan ${ }^{1, a}$, FengYunlling ${ }^{2, b}$ \\ ${ }^{1}$ QiaoNan, Textiles and Apparel Institute,Hebei University of Science and Technology,China \\ ${ }^{2}$ Feng Yunling, Fashion Institute, Hebei Academy of Fine Arts, China \\ aemail 519910777@qq.com, bemail 631537629@qq.com
}

Keywords: Nationalization Acrobatic garment design Wuqiao Innovation

Abstract: Wuqiao Acrobatics is famous for its distinctive local color,and is also one of the birthplace of the Chinese famous acrobatic art. Its acrobatic garment is based on the constant development and improvement of the folk clothing,with the growing influence of the "China Wuqiao International Acrobatics Art Festival"in the world acrobatics, the national innovation design of acrobatic garment is also very important.

Wuqiao is the birthplace of Chinese acrobatics art, known as the hometown of acrobatics, but also has a great influence on the development of the world's acrobatics . With the wide spread of the"China Wuqiao International Acrobatics Art Festival"in the international world of acrobatics, Wuqiao Acrobatics has gradually increased its international status not only been recognized in the world, but also to make the world a better understanding of the wisdom of the Chinese nation and culture. Today, as the first batch of national intangible cultural heritage of Wuqiao Acrobatics (the first country in 2006), is shouldering the responsibility of the spread of Chinese culture and art,not only on the rich and the creation of acrobatics, acrobatic garment in diversity combine fashion and nationality are also shoulder heavy responsibilities.

\section{Wuqiao Acrobatic garment}

Wuqiao acrobatics as a folk art with a long history, It not only has strength, skill and difficulty, but also integrate the many artistic characteristics of Chinese culture. Wuqiao acrobatics can become the birthplace of the Chinese acrobatics art, the hometown of acrobatics, which is inseparable with its features and artistic achievements, for its long history and "shock, dangerous, extraordinary, unique" truly artistic charm of the famous sea inside and outside, and its strong heritage and broad social influence can not be ignored. Wuqiao acrobatics rooted in folk, and their clothing which is same to Wuqiao acrobatics art, also inherited the tradition of folk garment, its distinctive folk culture, like a mirror, not only mapped out a side of modern and contemporary apparel development, but also enriches the connotation of Chinese clothing culture. Overall, acrobatic garment contains the head wear, foot wear and a variety of somatic clothing and also includes a comprehensive overall shape of acrobats modified decorative parts, such as hair, necklaces, pectoral, waist, arm ornaments, wristlet, foot ornaments, of course also including the actor's hair, makeup and so on, besides,design patterns, color matching and beads, embroidery is also an important part.

Development of Wuqiao Acrobatics garment.Wuqiao acrobatics garment from the life clothing, is on the basis of life clothing to enhance.We came to see acrobatics garment origin, purpose dressed first from practical, which is on the basis of daily clothing made to adapt to the needs of the action and performance of the changes, gradually formed its own unique structure and style features. 
Wuqiao Acrobatics garment development of modern basically divided into two levels: Charming low-level acrobatics garment which is life clothing and urban high-level acrobatics garment that is having a significant national characteristics, functionality and identification of performance clothing. Since the Song Dynasty, the prevalence among Wuqiao folk acrobatics, but acrobatics clothing is simple and plain which has nothing special style; It's different from walking in the countryside at the bottom of theartist, part of Wuqiao acrobatics artists in battles "Wild World" has been affirmed immediately after to the larger area and taken abroad.The changes in the performance of the stage to make the costume change, in addition to functionality, but they also demonstrate acrobatics clothing typical of traditional Chinese national characteristics ${ }^{[1]}$. The famous Wuqiao Acrobatics people Sunfengshan established "Beijing class" in 1926, when it came to Russia and the countries of Western Europe, the American continent and other regions show, the clothing worn on the ingenuity of the reform was designed to absorb the Chinese classical drama and martial arts clothing features:wearing the hat, wearing a martial arts warrior costumes, plus opera costume decorative embellishment, which is refreshing and popular with foreign visitors welcome. "Beijing class" acrobatic costume design, which is both approximately drama clothing and similar to Qing Dynasty Royal dress, it is orgeous bright in magnificent, but also reinforced the acrobatic performance is a representative of the modern Wuqiao acrobatics garment.

In modern times, the rapid development of modern acrobatics show a trend of specialization, high technology and high function. With the development of Wuqiao economic and acrobatics culture, acrobatic costume design standards are increasingly high,under the premise of ensuring the functional clothing, the characters began to need to pay attention to the clothing conforms to the characters and content, form, but also with the performance skills phase combined, absorb modern circus costume design, while fully focusing on the beauty of harmony and dress acrobatics dress ${ }^{[2]}$.

Geographical features of Wuqiao Acrobatics garment.Wuqiao is located in southeast of the junction of Jilu of Hebei Province, and from the perspective of the development of folk costumes, there are many things in common with the traditional clothing of farming civilization . Although the Wuqiao folk dress compared to affluent dress, style shape and Color decoration is more rustic decor seemed monotonous, but as ordinary people's dress has a typical characteristics of North China Plain and the mainland. This is not only due to its practicality, but also the adaptability of the environment, aesthetic and other factors. Wuqiao local folk costume has quite stable inheritance beyond the era, formed over a long historical period of their styles, patterns and cutting, art techniques, etc, remain the same or less long-term changes in the state.

Clothing acrobatics program in order to reflect its heritage character, folk elements are often used clothing directly or indirectly. Its acrobatics garment in order to reflect the program's heritage,folk elements are often used clothing directly or indirectly.The basic categories and decorative patterns of formerly Wuqiao acrobatics garment are derived from local folk costume Wuqiao belongs, the type, color, pattern and folk costume of the same strain, with a significant traditional cultural features. Garments no more than two basic shapes, shirt lower part and clothes shape, which is the most representative of the Chinese people clothing styles.

On women, the more wear more shirt lower part, the whole body of men wear robes, the two dressed shape sometimes used interchangeably, sometimes parallel development. Of course subdivision, coat also include tunic tops, half coat, jacket, shirt, vest, cloud shoulder, etc, under clothing including pants, skirts, such as chaps, phoenix-tail skirt, Pomegranate skirt,etc,some of the clothing are still worn by people in the folk. 


\section{Modernization and internationalization exemplification of Wuqiao acrobatics garment}

The development of foreign acrobatics garment.Modern acrobatic skills not only to complete the difficult moves, but also all kinds of art which must innovation fit to the arts. Let the audience get visual, auditory and mental aesthetic enjoyment in the process of viewing pleasure, so acrobatic fashion design has become closely associated with the program as a whole plastic arts ${ }^{[3]}$. Dominique McClure said: "Chinese acrobatics in technology has very high attainments, pay attention to the surprise and insurance, the action is very difficult, however, feels just gripped .While foreign acrobatics is very focused on the enjoyment of the audience's emotions, such as bright dress, the scene of the music, the clown's funny performance, can give a relaxed feeling".

In the international reputation of Canada's Cirque du Soleil as an example, in the program called "Half-Life", by just gorgeous with bright, green flash bionic scintillating acrobatic costume originality wins people's eyes: : great books to make props and rising cloud make audience be personally on the scene, their stage lighting and background design more people are flabbergasted, stirring. Clothing and accessories makeup is the Cirque du Soleil show in most attention of many elements, the designer's goal is to design a way to shape the role, so that the actors perform with ease and set in one of the beauty and safety clothing. Whether it is an angel in the balance of light dance, or the clown's weird humor of the play show a very high sense of innovation.Cirque du Soleil beautiful, skillful performance and the worldwide box office miracle, become one of the leading international acrobatics garment benchmark ${ }^{[4]}$.

The Situation of Wuqiao Acrobatics garment.With the continuous development of modern performing arts, people of acrobatics tasting ability has greatly improved. Audience is not only reading skills and performance, On the show all of the performance is to emphasize the role , are a factor of programming must be considered as a whole, of course, acrobatic costume is particularly important. Wuqiao Acrobatics garment modern fashion design gradually out a simple form of foreign plagiarism, but in general, artistic, creative and other aspects to explore, not only to meet the performers functional needs, but also suitable for theatrical content, sentiment, heighten the atmosphere of the show, highlighting the modern circus skills, art, variety and fashion ${ }^{[5]}$. The use of national characteristics, and the combination of modern fashion trends, so that the program has a strong visual impact and aesthetic appeal. Domestic famous acrobatic fashion designer Li Xiaomeng also emphasized, the most important point is that domestic acrobatic fashion design clothing should better reflect the national character, folk elements and geographical elements are to be used in acrobatic clever costume, which is modern acrobatic costume design a major feature.

Edado Moriero has been re-elected 15-year overall Monte Carlo International Circus Festival director ,he said: "Chinese acrobatics is different from Western acrobatics, props or costume sometimes more attractive than the program itself, and in the technology and performance, often with new elements, so Chinese acrobatics has been advanced in the world". Wuqiao acrobatics garment is also moving in the same direction as their skill,not only pay attention to reshape the traditional Chinese style, in the design process also pay more attention to detail, which based on the characteristics of fashion design combined with foreign acrobatics on the design ideas began to pluralism, rich and open, and the combination with experimental repertoire bold and innovative design, acrobatic costume to become the embodiment of an important complement of the acrobatic art value and aesthetic value, award-winning show, "Snake after waking - jujitsu" "Drum rhymeand "“spiritual - single used skills", in which costume fashion design are the successful example. 


\section{Nationalization innovation of Wuqiao Acrobatics garment}

The national spirit of Wuqiao acrobatics garment.Domestic and international acrobatic art of our times is different, Wuqiao Acrobatics how to gain a foothold in the world's forests, the important point is that the value of the Wuqiao Acrobatics reflect national character and national spirit, "nationalization" has become the first choice ${ }^{[6]}$. Wuqiao acrobatics has a history of thousands of years, $t$ in the development process of thick accumulation and reflects the strong national spirit and character of Yan Zhao is its unique charm, in Wuqiao Acrobatics modeled on the TV series "into the world", in which fully Wuqiao acrobats demonstrated the right way straight, self-improvement, love and hate, fear the strong, dare to fight, play a chivalrous, inclusive, pioneering and innovative spirit of Yanzhao.Acrobatic action programs not only demonstrate strong expression of national pride and superiority, but also to adapt to the development needs of its action acrobatic clothing (Wuqiao acrobatics is famous for its art highly difficult, many skills are based in martial arts), giving acrobatic costume with other performing arts clothing less some national characteristics.

National art features of Wuqiao acrobatics garment.From the comprehensive analysis of Wuqiao acrobatics clothing, we can see that the shape and style, techniques, clothing color, clothing accessories, decorative pattern and so on are reflected in the Wuqiao folk art characteristics, its connotation is the inheritance of Wuqiao folk costume content. With the development of modern Wuqiao Acrobatics and rich, Wuqiao Acrobatics clothing is also updated with the times changing, showing unprecedented prosperity.

Nationalization of Wuqiao Acrobatics kind of garment styles.Wuqiao acrobatics clothing style is a lot of traditional folk clothing on the basis of re design performance. As children's acrobatic show "light coupling", the performance of Chinese traditional festival, "Lantern Festival", mainly young performers, in order to highlight a child's innocence and active, "Apron" has become prominent typical images of choice, through appropriate content and performance re-design operation, fully demonstrated the program creative deep meaning and traditional folk culture ${ }^{[7]}$. Again, upside down and kicking skills class clothing are designed, using the original folk pants, but in cuffs, knees, waist Department and other activities are carried out in accordance with specific programs to facilitate the performance of the details of the design process, but also the whole actor dress on the implication of a unique effect.

Nationalization of Wuqiao Acrobatics garment color.Color is the first thing part of people's eyes, is the most accessible part of prominent national characteristics, but also is combined with the most modern aesthetic embodies the fashion part. Wuqiao Acrobatics garment color aspect of the use of the common folk of the northern plain colors and contrasting colors, on the other hand,weakening of the purity increases the overall sense of tone and the gray leve, is common practice in national style design. For example, red and yellow tones diminish its purity and make it increasingly stable, condensed, blue and green tones also more emphasis on coordination with contrasting colors, by adding color transition, including color gray and center, with the overall harmony, local contrast, reflecting the unadorned and generous, palatial designarrangement is the national costume of good performance.

Wuqiao Acrobatics garment color, followed the Wuqiao folk clothing of Han nationality colors habits, rich and gaudy colors, with great ornamental value and symbolic meaning, the most prominent of the character and customs of the local people's Wuqiao warrior of regional culture character and bold straightforward custom character. "Favorite red" is the Wuqiao Acrobatics garment colors typical of a feature, "Favorite red" mentality among Wuqiao folk social life also has a deep meaning: on the one hand,it is Similar to the Chinese favorite red psychological consistency, 
meaning auspiciousness; on the other hand, it is also related with magnificent and pessimistic ,chivalrous play Yanzhao strength of character, especially in some martial arts skills in acrobatics will often appear. Wuqiao Acrobatics garment inherited a strong color contrast the traditional characteristics, but also related to acrobatics with shock, dangerous, extraordinary, new features , use of bright, pleasing, exciting and lively colors, decorative and identified by the color of the configuration, highlighting its strong and dense rich beauty. Acrobatics show "jujitsu rolling light" clothing is more in red tone, ingenious yellow, purple, blue, green and other colors, making a great impression on the whole harmony.

The application of Hebei folk decoration technique in Wuqiao acrobatics garment.Paper cutting- as Hebei folk tradition of decorative techniques, are often used in Wuqiao Acrobatics garment.Wuqiao circus troupe performances of the "Festival - women's modeling" program, patterned on its clothing is the use of paper-cutting practices. White fabric to make clothing body, in the chest and leg side armpit use red paper-cut butterfly pattern dressing, fresh and elegant in red and white, full of national characteristics. White fabric to make clothing body, use red paper butterfly pattern decorative embellishment in the chest armpit and leg side, fresh and elegant in red and white, full of national characteristics.

Embroidery as Wuqiao one of the most popular folk decoration, has a long history andoutstanding achievements in art,Embroidery on Wuqiao acrobatics clothing has a wide variety of techniques, mainly from Wuqiao folk weave, embroider, pick, dyeing and post traditional embroidery craft, its design simplicity, simple composition, shape exaggeration, mostly to Antique embroidered, each to a grand festival occasions, acrobats wear ornate costume performing acrobatics show, on which a variety of clothing embroidery techniques, reflecting the people's desire for a better life. Wuqiao acrobatics flourished when the old development, both in the fabric weave embroidered directly added, there are also decorative embroidery patterns on the plain, in order to fresh and elegant, so that embroidery has become the clothing patterns reflect the important technical means, therefore,patterns and embroidery on the acrobatics garment are complementary. While modern, due to the low efficiency of manual embroidery,slow and can not keep up the pace of acrobatic costume update, so traditional folk embroidery, often by way of embroidery in modern acrobatics garment decoration reflected ${ }^{[8] \text {. }}$

Wuqiao acrobatics garment pattern is rich and colorful,they are mostly drawn from the animals, mountains, flowers, birds, poultry, fish and insects. Acrobatic costume pattern of main body is the dragon and Phoenix, such as dragon and Phoenix, flamboyant, Kowloon playing with a pearl, Dilophosaurus downtown fungus and other grain map, not only is a metaphor for the totem worship , and represents the symbol of the whole spirit of Chinese culture. In the modern acrobatic costume design often through special printing techniques (such as gold and silver powder, pearl powder, dimmer, color printing, etc.), decorative beads, decorative collage and other techniques, the clever use of these patterns in a variety of materials, and enhance beauty of acrobatics garment and contrast performance combined with choreography atmosphere ${ }^{[9] .}$

\section{Conclusions(The future development and Prospect of Wuqiao acrobatics garment)}

When we went to look at the dynamic acrobatic art from the perspective of clothing, you can feel the importance of clothing Portrayal of acrobatics arts. As art and technology combined with the product, acrobatics costume design is acrobatic art development of witness and the changes of the times of the pulse beating,to some extent reflects the culture of the country and part of a national spirit. In the current trend of globalization, acrobatics costume design must have its own national identity, and into the fashion element, performers and repertoire, skills, background, lighting, 
choreography and choreography are closely linked, so that it can in the field of international acrobatics art in perpetuity. North Korean Acrobatics Troupe Colonel Jin Chuangzhu said that the technical performance of acrobatics to have their own national characteristics, should be in accordance with their own national style to develop, in order to the future on the road to flash. As a whole clothing acrobatic show is true, the future design perspective Wuqiao Acrobatics clothing will move toward a more prominent characteristics of the direction of nationalization, not only embodies the program in the plot, viewing pleasure and aesthetic fashion, while also presenting innovative programs, regional and nationality and so on.

In short, an important trend in the 21 st century circus costume is great importance to the nation and the interpretation of traditional shape, with an emphasis on design details and modern languages. Spirit of national culture and ideas to modernize the interpretation, looking for inspiration from the traditional arts and culture and create a useful, has become an important development direction of contemporary fashion design and acrobatics clothing.

\section{References}

[1] Yanju Cao,Changes feature of Wuqiao Acrobatics clothing ,Textile Journal,06,2007.

[2] Fengqin Hu, Acrobatic costume features and design factor,Industry sketches and notes,02,2013.

[3] Fengyu Yang,The main points of the costume design analysis,Acrobatics and magic,06,2012.

[4] $\mathrm{Xu}$ Chen,Talking about the design,production and personnel training acrobatics clothing,Lamp sketches and notes, 10,2008.

[5] Xun Zhou.Chunming Gao,Chinese ancient costumes,Shanghai: Academia Press, 1996.

[6] Yan Li,Acrobatics clothing creativity and performance,Industry sketches and notes,08,2010.

[7] Yanju Cao,Origin relationship with the local folk Wuqiao Acrobatics clothing,Academic Papers,03,2008.

[8] Yan Li,Acrobatics clothing creativity and performance,Industry sketches and notes,08,2010.

[9] Yanju Cao,Clothing nationalization Perspective design,Luohe Vocational and Technical College,07,2013. 